\title{
An inequality for the gamma function via statistics and applications
}

Pingping Zhang and Mingjin Wang*

\section{"Correspondence:} wang197913@126.com

Department of Mathematics, Changzhou University, Changzhou, Jiangsu 213164, P.R. China

\begin{abstract}
The aim of this paper is to establish an inequality for the gamma function, using a statistical method. Applications of the inequality are also given, including some estimates of $\pi$.
\end{abstract}

MSC: Primary 62F11;33B15;33B10

Keywords: inequality; variance; unbiased estimate; UMVUE

\section{Introduction and main result}

Recently, there have been many papers about the ratio of gamma functions in the literature; see [1-9]. Some of the papers use statistical methods. Gurland [10] has given an inequality satisfied by the gamma function, using the so-called Cramér-Rao lower bound for the variance of unbiased estimators. Olkin [11] has given an extension of Gurland's inequality. Gokhale [12] has given another inequality, which used an analogue of the CramérRao lower bound derived by Rao [13]. Rao gave a stronger version of Wallis' formula [14]. We, inspired by the above papers, give an inequality concerning the gamma function. Applications of the inequality are also given. We first recall some definitions, notation, and well-known results in statistical theory, which will be used in this paper.

A normal distribution $N\left(\mu, \sigma^{2}\right)$ is described by the probability density function,

$$
p(x)=\frac{1}{\sqrt{2 \pi} \sigma} e^{-\frac{(x-\mu)^{2}}{2 \sigma^{2}}}, \quad x \in \mathbb{R}
$$

When a random variable $X$ is distributed normally with mean $\mu$ and variance $\sigma^{2}$, we write $X \sim N\left(\mu, \sigma^{2}\right)$.

Suppose that $x_{1}, x_{2}, \ldots, x_{n}$ is a sample from a population with a distribution function $F_{\theta}(x)(\theta \in \Omega)$. Let $\hat{g}=\hat{g}\left(x_{1}, x_{2}, \ldots, x_{n}\right)$ be an estimator of a parametric function $g(\theta)$. If $E(\hat{g})=$ $g(\theta)$ for all values of parameter $\theta \in \Omega$, we call $\hat{g}$ an unbiased estimator of $g(\theta)$.

Consider an estimation of $g(\theta)$ based on a sample $x_{1}, x_{2}, \ldots, x_{n}$ from some member of a family of distribution functions $F_{\theta}(x), \theta \in \Omega$, where $\Omega$ is the parameter space. An unbiased estimator $\hat{g}\left(x_{1}, x_{2}, \ldots, x_{n}\right)$ of $g(\theta)$ is UMVUE, if $\forall \theta \in \Omega$,

$$
\operatorname{var}_{\theta}\left(\hat{g}\left(x_{1}, x_{2}, \ldots, x_{n}\right) \leq \operatorname{var}_{\theta}\left(\tilde{g}\left(x_{1}, x_{2}, \ldots, x_{n}\right)\right)\right.
$$

for any other unbiased estimator $\tilde{g}$.

(c) 2015 Zhang and Wang. This article is distributed under the terms of the Creative Commons Attribution 4.0 International License (http://creativecommons.org/licenses/by/4.0/), which permits unrestricted use, distribution, and reproduction in any medium, provided you give appropriate credit to the original author(s) and the source, provide a link to the Creative Commons license, and indicate if changes were made. 
Euler's gamma function $\Gamma$ is defined for $x>0$ by

$$
\Gamma(x)=\int_{0}^{\infty} t^{x-1} e^{-t} d t .
$$

If $x_{1}, x_{2}, \ldots, x_{n}$ is a sample from a population with distribution $N\left(\mu, \sigma^{2}\right)$, then

$$
\hat{\sigma}=\frac{\Gamma\left(\frac{n}{2}\right)}{\sqrt{2} \Gamma\left(\frac{n+1}{2}\right)} \sqrt{\sum_{i=1}^{n} x_{i}^{2}}
$$

is the UMVUE of $\sigma$.

The main result of this paper is the following theorem.

Theorem 1.1 Suppose that $n_{k}(k=1,2, \ldots, m)$ are nonnegative integers and $\lambda_{k} \in \mathbb{R}$, such that $0 \leq \lambda_{k} \leq 1, \sum_{k=1}^{m} \lambda_{k}=1$. Then we have

$$
\frac{n \Gamma^{2}\left(\frac{n}{2}\right)}{2 \Gamma^{2}\left(\frac{n+1}{2}\right)}-1 \leq \sum_{k=1}^{m} \lambda_{k}^{2}\left(\frac{n_{k} \Gamma^{2}\left(\frac{n_{k}}{2}\right)}{2 \Gamma^{2}\left(\frac{n_{k}+1}{2}\right)}-1\right),
$$

where $n=\sum_{k=1}^{m} n_{k}$.

\section{Proof of the main result}

In this section, we use statistical methods to prove the theorem.

Proof Let $x_{11}, x_{12}, \ldots, x_{1 n_{1}}, x_{21}, x_{22}, \ldots, x_{2 n_{2}}, x_{m 1}, x_{m 2}, \ldots, x_{m n_{m}}$ be a random sample from a normal distribution $X \sim N\left(\mu, \sigma^{2}\right)$. From (1.4), it is known that

$$
\hat{\sigma}=\frac{\Gamma\left(\frac{n}{2}\right)}{\sqrt{2} \Gamma\left(\frac{n+1}{2}\right)} \sqrt{\sum_{i=1}^{m} \sum_{j=1}^{n_{i}} x_{i j}^{2}}
$$

is the UMVUE of $\sigma$, where $n=\sum_{i=1}^{m} n_{i}$.

For any $x_{k 1}, x_{k 2}, \ldots, x_{k n_{k}}, 1 \leq k \leq m$

$$
\frac{\Gamma\left(\frac{n_{k}}{2}\right)}{\sqrt{2} \Gamma\left(\frac{n_{k}+1}{2}\right)} \sqrt{\sum_{k=1}^{n_{k}} x_{k i}^{2}}
$$

is an unbiased estimate of $\sigma$.

Using (2.2), we construct a new unbiased estimate of $\sigma$, i.e.,

$$
\hat{\sigma}_{1}=\sum_{k=1}^{m} \frac{\lambda_{k} \Gamma\left(\frac{n_{k}}{2}\right)}{\sqrt{2} \Gamma\left(\frac{n_{k}+1}{2}\right)} \sqrt{\sum_{k=1}^{n_{k}} x_{k i}^{2}},
$$

where $0 \leq \lambda_{k} \leq 1, \sum_{k=1}^{m} \lambda_{k}=1$.

Due to the definition of the UMVUE, the following inequality holds:

$$
\operatorname{Var} \hat{\sigma}_{1} \geq \operatorname{Var} \hat{\sigma}
$$


After some simple computations, we can obtain

$$
D(\hat{\sigma})=\left(\frac{n \Gamma^{2}\left(\frac{n}{2}\right)}{2 \Gamma^{2}\left(\frac{n+1}{2}\right)}-1\right) \sigma^{2}
$$

and

$$
D\left(\hat{\sigma}_{1}\right)=\sum_{k=1}^{m} \lambda_{k}^{2}\left(\frac{n_{k} \Gamma^{2}\left(\frac{n_{k}}{2}\right)}{2 \Gamma^{2}\left(\frac{n_{k}+1}{2}\right)}-1\right) \sigma^{2} .
$$

Substituting (2.5) and (2.6) into (2.4) gives (1.5). Thus, we complete the proof.

\section{Some applications of Theorem 1.1}

In this section, we show some applications of the main result of this paper. First, we give the following inequalities, which include the gamma function and a trigonometric function.

Theorem 3.1 Suppose $n$ is any positive integer, and $\theta \in \mathbb{R}$, then

$$
\frac{\Gamma^{2}(2 n+1)}{\Gamma^{2}\left(2 n+\frac{1}{2}\right)} \leq n \sin ^{2} \theta+\left(2-\sin ^{2} \theta\right) \frac{\Gamma^{2}(n+1)}{\Gamma^{2}\left(n+\frac{1}{2}\right)}
$$

Proof Letting $m=2$ and $\lambda_{1}=\sin ^{2} \theta, \lambda_{2}=\cos ^{2} \theta$ in (1.5) gives

$$
\begin{aligned}
& \frac{\left(n_{1}+n_{2}\right) \Gamma^{2}\left(\frac{n_{1}+n_{2}}{2}\right)}{2 \Gamma^{2}\left(\frac{n_{1}+n_{2}+1}{2}\right)}-1 \\
& \quad \leq \sin ^{4} \theta\left(\frac{n_{1} \Gamma^{2}\left(\frac{n_{1}}{2}\right)}{2 \Gamma^{2}\left(\frac{n_{1}+1}{2}\right)}-1\right)+\cos ^{4} \theta\left(\frac{n_{2} \Gamma^{2}\left(\frac{n_{2}}{2}\right)}{2 \Gamma^{2}\left(\frac{n_{2}+1}{2}\right)}-1\right) .
\end{aligned}
$$

Letting $n_{1}=n_{2}=a$ in (3.2), one obtains

$$
\frac{\Gamma^{2}(2 a+1)}{\Gamma^{2}\left(2 a+\frac{1}{2}\right)}-2\left(\sin ^{4} \theta+\cos ^{4} \theta\right) \frac{\Gamma^{2}(a+1)}{\Gamma^{2}\left(a+\frac{1}{2}\right)} \leq 2 a\left(1-\sin ^{4} \theta-\cos ^{4} \theta\right) .
$$

Employing the following trigonometric formula:

$$
\sin ^{4} \theta+\cos ^{4} \theta=1-\frac{\sin ^{2} 2 \theta}{2}
$$

(3.3) becomes

$$
\frac{\Gamma^{2}(2 a+1)}{\Gamma^{2}\left(2 a+\frac{1}{2}\right)} \leq a \sin ^{2}(2 \theta)+\left(2-\sin ^{2}(2 \theta)\right) \frac{\Gamma^{2}(a+1)}{\Gamma^{2}\left(a+\frac{1}{2}\right)} .
$$

Replacing $2 \theta$ by $\theta$ and $a$ by $n$, we obtain (3.1). Thus, we finish the proof.

In [15], Gurland gave the following estimator of $\pi$ :

$$
\frac{4 n+3}{(2 n+1)^{2}}\left(\frac{(2 n) ! !}{(2 n-1) ! !}\right)^{2}<\pi .
$$


Mortici [1] gave the refinements of Gurland's formula for $\pi$ :

$$
\left(\frac{n+\frac{1}{4}}{n^{2}+\frac{1}{2} n+\frac{3}{32}}+\frac{9}{2,048 n^{5}}-\frac{45}{8,192 n^{6}}\right)\left(\frac{(2 n) ! !}{(2 n-1) ! !}\right)^{2}<\pi
$$

Using (3.1), we can get the following similar result.

Corollary 3.2 Suppose $n$ is any nonnegative integer, then

$$
\frac{1}{n}\left[\left(\frac{(4 n) ! !}{(4 n-1) ! !}\right)^{2}-\left(\frac{(2 n) ! !}{(2 n-1) ! !}\right)^{2}\right]<\pi
$$

Proof Letting $\theta=\frac{\pi}{2}$ in (3.1) gives

$$
\frac{\Gamma^{2}(2 n+1)}{\Gamma^{2}\left(2 n+\frac{1}{2}\right)} \leq n+\frac{\Gamma^{2}(n+1)}{\Gamma^{2}\left(n+\frac{1}{2}\right)}
$$

We have

$$
\frac{(2 q) ! !}{(2 q-1) ! !}=\sqrt{\pi} \frac{\Gamma(q+1)}{\Gamma\left(q+\frac{1}{2}\right)} .
$$

See, e.g., [1]. So

$$
\frac{1}{n}\left[\left(\frac{(4 n) ! !}{(4 n-1) ! !}\right)^{2}-\left(\frac{(2 n) ! !}{(2 n-1) ! !}\right)^{2}\right] \leq \pi .
$$

Because the equality in (3.11) cannot hold, we get (3.8).

Now we give an inequality involving combinational coefficients $\left(\begin{array}{l}n \\ m\end{array}\right)$, defined by

$$
\left(\begin{array}{c}
n \\
m
\end{array}\right)=\frac{n !}{m !(n-m) !}
$$

Theorem 3.3 Suppose $m, w$ are any positive integers, then

$$
\begin{aligned}
& \frac{1}{m}\left[\frac{(2 m w) ! !}{(2 m w-1) ! !}\right]^{2}+\frac{1}{2^{2 m}}\left[w \pi-\left(\frac{(2 w) ! !}{(2 w-1) ! !}\right)^{2}\right]\left[\left(\begin{array}{c}
2 m \\
m
\end{array}\right)-1\right] \\
& \quad \leq w \pi
\end{aligned}
$$

Proof Since

$$
\sum_{k=1}^{m}\left(\begin{array}{l}
m \\
k
\end{array}\right) \frac{1}{2^{m}}=1
$$

Letting $n_{k}=2 w, \lambda_{k}=\left(\begin{array}{c}m \\ k\end{array}\right) \frac{1}{2^{m}}$ in (1.5), then $n=\sum_{k=1}^{m} n_{k}=2 m w$. We get

$$
m w \frac{\Gamma^{2}(m w)}{\Gamma^{2}\left(m w+\frac{1}{2}\right)}-1 \leq \sum_{k=1}^{m}\left(\left(\begin{array}{c}
m \\
k
\end{array}\right) \frac{1}{2^{m}}\right)^{2}\left(w \frac{\Gamma^{2}(w)}{\Gamma^{2}\left(w+\frac{1}{2}\right)}-1\right) .
$$


Using the inequality of (3.10) and after some simple derivations, we have

$$
\begin{aligned}
& {\left[\frac{1}{m}\left(\frac{(2 m w) ! !}{(2 m w-1) ! !}\right)^{2}-\sum_{k=1}^{m}\left(\left(\begin{array}{c}
m \\
k
\end{array}\right) \frac{(2 w) ! !}{(2 w-1) ! !} \frac{1}{2^{m}}\right)^{2}\right] \frac{1}{\pi}} \\
& \quad \leq w\left[1-\sum_{k=1}^{m}\left(\left(\begin{array}{c}
m \\
k
\end{array}\right) \frac{1}{2^{m}}\right)^{2}\right] .
\end{aligned}
$$

Substituting

$$
\sum_{k=0}^{m}\left(\begin{array}{c}
m \\
k
\end{array}\right)^{2}=\left(\begin{array}{c}
2 m \\
m
\end{array}\right)
$$

into (3.15) one obtains (3.12).

The special case $w=1$ of (3.12) results in

$$
\frac{1}{m}\left[\frac{(2 m) ! !}{(2 m-1) ! !}\right]^{2}+\frac{\pi-4}{2^{2 m}}\left[\left(\begin{array}{c}
2 m \\
m
\end{array}\right)-1\right] \leq \pi
$$

Finally, we give the following double inequality for $\pi$.

Theorem 3.4 Let $p, d$ are positive integers, then

$$
\begin{aligned}
\frac{1}{2 p d} & {\left[\left(\frac{(4 p d+2 p) ! !}{(4 p d+2 p-1) ! !}\right)^{2}-\left(\frac{(2 p) ! !}{(2 p-1) ! !}\right)^{2}\right] } \\
\quad<\pi & <\frac{4 d}{(2 p+1)\left[(2 d+1)^{2}\left(\frac{(4 p d+2 p+2 d-1) ! !}{(4 p d+2 p+2 d) ! !}\right)^{2}-\left(\frac{(2 p-1) ! !}{(2 p) ! !}\right)^{2}\right]} .
\end{aligned}
$$

Proof Letting $m=2 d+1$ and $n_{1}=n_{2}=\cdots=n_{m}=2 p+1$ in (1.5) gives

$$
\begin{aligned}
& \frac{(2 p+1)(2 d+1)}{2} \frac{\Gamma^{2}\left(2 p d+p+d+\frac{1}{2}\right)}{\Gamma^{2}(2 p d+p+d+1)}-1 \\
& \leq \sum_{k=1}^{2 d+1} \lambda_{k}^{2}\left[\frac{2 p+1}{2} \frac{\Gamma^{2}\left(\frac{2 p+1}{2}\right)}{\Gamma^{2}\left(\frac{2 p+2}{2}\right)}-1\right] .
\end{aligned}
$$

Using (3.10), the inequality (3.19) can be written in the equivalent form

$$
\pi<\frac{2\left(1-\sum_{k=1}^{2 d+1} \lambda_{k}^{2}\right)}{(2 p+1)\left[(2 d+1)\left(\frac{(4 p d+2 p+2 d-1) !}{(4 p d+2 p+2 d) ! !}\right)^{2}-\sum_{k=1}^{2 d+1} \lambda_{k}^{2}\left(\frac{(2 p-1) ! !}{(2 p) ! !}\right)^{2}\right]} .
$$

Letting $\lambda_{1}=\lambda_{2}=\cdots=\lambda_{m}=\frac{1}{2 d+1}$ in (3.20) gives

$$
\pi<\frac{4 d}{(2 p+1)\left[(2 d+1)^{2}\left(\frac{(4 p d+2 p+2 d-1) ! !}{(4 p d+2 p+2 d) ! !}\right)^{2}-\left(\frac{(2 p-1) ! !}{(2 p) ! !}\right)^{2}\right]} .
$$


Similarly, letting $m=2 d+1, n_{1}=n_{2}=\cdots=n_{m}=2 p$, and $\lambda_{1}=\lambda_{2}=\cdots=\lambda_{m}=\frac{1}{2 d+1}$ in (1.5), one can obtain

$$
\frac{1}{2 p d}\left[\left(\frac{(4 p d+2 p) ! !}{(4 p d+2 p-1) ! !}\right)^{2}-\left(\frac{(2 p) ! !}{(2 p-1) ! !}\right)^{2}\right]<\pi .
$$

Then the inequality of (3.18) is the combination of the inequality (3.21) and the inequality (3.22).

The special case $p=1$ of (3.18) results in

$$
\frac{1}{2 d}\left[\left(\frac{(4 d+2) ! !}{(4 d+1) ! !}\right)^{2}-4\right]<\pi<\frac{4 d}{3\left[(2 d+1)^{2}\left(\frac{(6 d+1) ! !}{(6 d+2) ! !}\right)^{2}-\frac{1}{4}\right]} .
$$

\section{Competing interests}

The authors declare that they have no competing interests.

\section{Authors' contributions}

All authors read and approved the final manuscript.

\section{Acknowledgements}

The authors would like to thank two anonymous referees for many helpful comments and suggestions. We acknowledge support by the National Natural Science Foundation (grant 11271057) of China.

Received: 23 December 2014 Accepted: 20 May 2015 Published online: 10 June 2015

\section{References}

1. Mortici, C: Refinements of Gurland' formula for pi. Comput. Math. Appl. 62, 2616-2620 (2011)

2. Mortici, C: New approximation formulas for evaluating the ratio of gamma functions. Math. Comput. Model. 52(1), 425-433 (2010)

3. Mortici, C: Sharp inequalities and complete monotonicity for the Wallis ratio. Bull. Belg. Math. Soc. Simon Stevin 17(5), 929-936 (2010)

4. Mortici, C, Qi, F: Some best approximation formulas and inequalities for the Wallis ratio. Appl. Math. Comput. 253, 363-368 (2015)

5. Mortici, C, Lu, D, Cristea, V: Complete monotonic functions and inequalities associated to some ratio of gamma function. Appl. Math. Comput. 240, 168-174 (2014)

6. Qi, F: Bounds for the ratio of two gamma functions: from Gautschi's and Kershaw's inequalities to complete monotonicity. Turk. J. Anal. Number Theory 2(5), 152-164 (2014). doi:10.12691/tjant-2-5-1

7. Qi, F, Luo, Q-M: Bounds for the ratio of two gamma functions: from Wendel's asymptotic relation to Elezović-Giordano-Pečarić's theorem. J. Inequal. Appl. 2013, 542 (2013). doi:10.1186/1029-242X-2013-542

8. Qi, F, Luo, Q-M: Bounds for the ratio of two gamma functions - from Wendel's and related inequalities to logarithmically completely monotonic functions. Banach J. Math. Anal. 6(2), 132-158 (2012)

9. Qi, F: Bounds for the ratio of two gamma functions. J. Inequal. Appl. 2010, Article ID 493058 (2010). doi:10.1155/2010/493058

10. Gurland, J: An inequality satisfied by the gamma function. Skand. Aktuarietidskr. 1956, 171-172 (1956)

11. Olkin, I: An inequality satisfied by the gamma function. Skand. Aktuarietidskr. 1959, 37-39 (1959)

12. Gokhale, DV: On an inequality for gamma functions. Skand. Aktuarietidskr. 1962, 213-215 (1962)

13. Rao, BR: On an analogue of Cramer-Rao inequality. Skand. Aktuarietidskr. 1959, 213-215 (1959)

14. Rao Uppuluri, VR: On a stronger version of Wallis' formula. Pac. J. Math. 19(1), 183-187 (1966)

15. Gurland, J: On Wallis' formula. Am. Math. Mon. 63, 643-645 (1956) 